\title{
Graded home introduction of egg is safe, well received and cost effective when managing childhood egg allergy
}

\author{
Leah Thomas ${ }^{1}$, Jan Belcher ${ }^{1}$, Rachael Phillips ${ }^{1}$, Kahn Preece ${ }^{1}$, and Rani Bhatia ${ }^{1}$ \\ ${ }^{1}$ John Hunter Children's Hospital
}

April 7, 2021

Graded home introduction of egg is safe, well received and cost effective when managing childhood egg allergy

To the Editor,

In Australia, an IgE mediated allergy to egg is a common food allergy in children. ${ }^{1-2}$ The 2011 HealthNuts study determined that $8.9 \%$ of children in Australia have an egg allergy. ${ }^{1-2} \operatorname{IgE}$ mediated allergy to egg can be mild and most children may outgrow this by $4-5$ years of age. ${ }^{3-4}$ Almost $80 \%$ of children with IgE mediated egg allergy are tolerant of egg in a baked form with wheat being used as a matrix. ${ }^{1,3}$ Turner et al. suggests that the outcome of baked egg challenges can be unpredictable and can lead to anaphylaxis even in children with prior mild symptoms. ${ }^{5}$ Tolerance to baked egg is generally confirmed by a baked egg food challenge in hospital after which patients are encouraged to introduce baked egg products at home. ${ }^{6}$ Patients are reassessed and subsequently undergo a lightly cooked egg challenge in hospital where resolution is confirmed if successful. ${ }^{6}$

Whereas there is evidence that majority of children with an egg allergy are tolerant to baked egg, there is debate as to whether it can reduce the duration of egg allergy. ${ }^{7-9}$ Recently, Gotesdyner et al found that a structured graded exposure using baked egg followed by lightly cooked egg compared to complete avoidance helped achieve tolerance to egg. ${ }^{3}$ Their findings suggest that an egg ladder may promote a faster resolution of egg allergy. However, due to the case control study design and small sample size definite conclusions could not be drawn. ${ }^{3}$

Our Paediatric Allergy Service is a busy tertiary level clinic with more than 2000 outpatient interactions annually. To reduce the burden on tertiary resources, an egg tolerance ladder was developed (Appendix 1). It has been offered to patients considered low risk which includes (but is not limited to) patients with a single food allergy; mild or no eczema; mild, well controlled or no coexisting asthma; and/or a history of IgE mediated egg allergy without anaphylaxis. It has been designed to allow slow introduction of baked egg followed by foods containing small amounts of cooked egg and finally lightly cooked egg at home. Support throughout the home introduction process is provided by the treating team via email and phone contact.

The aim of this study was to evaluate the use of this structured "egg ladder" with regards to its safety as well as user satisfaction and barriers that arose in negotiating it. We also attempted to determine potential risk factors which increased likelihood of clinical reaction to foods containing egg and rates of eventual tolerance to lightly cooked and raw egg achieved in the home environment. This study was approved by the Ethics Committee at John Hunter Children's Hospital (2020/ETH01192).

Patients with mild to moderate IgE mediated egg allergy aged 0-18 years seen in the Paediatric Allergy Clinic and commenced on an egg tolerance ladder by the Paediatric Immunologist were recruited retrospectively. Verbal and written education on the use of the ladder had been provided at the time of clinic review. Patients 
were excluded from home introduction if they had a history of anaphylaxis to any food containing egg or a non-IgE mediated egg allergy.

A file audit identified a total of 98 patients for inclusion in this study who had been commenced on the egg ladder between September 2018 and June 2020. Patients were contacted by email and phone, and offered participation in a telephone survey to evaluate the use of the egg tolerance ladder. Almost half (47 patients) consented to the study. At the time of commencing the egg ladder, patients had a mean age of 40 months (IQR: 12-60 months). Six children did not have a skin prick test (SPT). The majority were sensitised with a mean SPT of $3.1 \mathrm{~mm}$. Most (66\%) had at least one atopic comorbidity and almost half had more than one food allergy. The vast majority $(87.2 \%)$ of patients commenced the egg ladder at home. This includes 23 patients already tolerant to baked egg prior to clinic presentation, based on clinical history. Only 3 (6\%) used the resources of an inpatient challenge.

Patients had spent an average of 15.5 months (IQR: 9-21.5 months) on the ladder. At the time of review, $43 \%$ of patients had completed the egg ladder but interestingly, four parents believed their child was still allergic to egg. The mean age of commencement on the egg ladder was higher than expected ( 40 months), and around the age a child is expected to outgrow an allergy to egg. ${ }^{3-4}$ Despite this, many patients reported reactions while using the egg ladder. A mild reaction was reported by $18(38 \%)$ parents, 16 required treatment and of those one was given adrenaline. This child was 6 years of age, had an isolated egg allergy and small skin test (3.5mm). They reacted to Step 5 and have continued Step 4 at home successfully. Both families reporting a severe reaction recommenced the ladder and were able to subsequently progress successfully to Step 5 or 6 . The majority had a mild skin response (rash or hives). Skin testing was a poor differentiator for clinical reaction with $38 \%$ of those with negative skin testing reporting a mild reaction with baked egg.

Parental satisfaction was high with $78.7 \%$ satisfied or very satisfied with the egg ladder. Most of the remaining families were neutral with no families dissatisfied with the ladder use. Many stated that they valued the structured approach of introducing egg which helped identify their child's level of tolerance, and allowed them to progress at their own pace. A number of parents identified the main barrier to progressing the ladder was the taste and/or texture of egg after successfully completing the ladder and subsequent difficulty maintaining regular lightly cooked egg in the diet. These families continued egg as an ingredient in cooked or baked food.

There were some limitations to this study. Firstly, this was a retrospective small sized survey and some patients were unable to accurately recall timelines. As a voluntary survey there is also a risk of ascertainment bias. Baseline patient demographics (age, sex, SPT size, comorbid allergy diagnoses) of non-responders were compared to the study cohort with no significant differences identified. The initial education on commencing on the egg ladder was provided by multiple clinicians and information therefore was unable to be standardised. As expected, a number of patients at the time of the survey were still navigating the egg ladder and therefore had not had enough time to reach their individual threshold to determine if their child had outgrown their allergy. This may lead to under-reporting of egg tolerance on the ladder. Finally, follow up or assistance with the egg ladder was not provided unless the parent contacted the team. This may have resulted in less children completing the ladder due to parental anxiety over mild reactions.

We have shown that the use of a structured egg ladder for egg allergic children without a history of anaphylaxis can be safe, is well tolerated and is a positive experience for families. It can prevent time consuming and costly inpatient supervised challenges unnecessarily consuming valuable and scarce resources in busy allergy clinics. Future prospective studies will help establish the role of home egg introduction in childhood egg allergy management in both tertiary and secondary health care settings.

Conflicts of Interest

No conflicts to be declared.

Leah Thomas ${ }^{1}$

Jan Belcher ${ }^{1}$ 
Rachael Phillips ${ }^{1}$

Kahn Preece, MBBS, FRACP ${ }^{1}$

Rani Bhatia, MBBS, FRACP ${ }^{1}$

${ }^{1}$ Department of Paediatric Allergy and Immunology, John Hunter Children's Hospital, Newcastle, Australia

References

1. Osborne NJ, Koplin JJ, Martin PE, et al. Prevalence of challenge-proven IgE-mediated food allergy using population-based sampling and predetermined challenge criteria in infants. Journal of Allergy and Clinical Immunology. 2011;127:668-76.

2. Dang TD, Peters RL, Koplin JJ, et al. Egg allergen specific IgE diversity predicts resolution of egg allergy in the population cohort HealthNuts. Allergy. 2019; 74: 2.

3. Gotesdyner L, Zeldin Y et al. A structured graduated protocol with heat denatured eggs in the treatment of egg allergy. Pediatric Allergy and Immunology. 2019;30:824-832.

4. Leonard SA, Sampson HA, Sicerer SH, et al. Dietary baked egg accelerates resolution of egg allergy in children. Journal of Allergy and Clinical Immunology. 2012;13:2.

5. Turner PJ, Mehr S, Joshi P et al. Safety to food challenges to extensively heated egg in egg-allergic children: a prospective cohort study. Pediatric Allergy and Immunology. 2013;24:450-455.

6. Clark A, Islam S, King Y. A longitudinal study of resolution of allergy to well-cooked and uncooked egg. Clinical \& Experimental Allergy. 2011;41:706-712.

7 Lambert R, Grimshaw K, Elis B et al. Evidence that eating baked egg or milk influences egg or milk allergy resolution: A Systematic Review. Clin Exp Allergy. 2017;47(6):829-837.

8. Upton J, Nowak-Wegrzyn A. The impact of baked egg and baked milk diets on IgE and Non-IgE mediated allergy. Clinical Reviews in Allergy \& Immunology. 2018;55(2):118-138.

9. Leonard SA, Caubet JC, Kim, JS, Groetch M, Wegrzyn AN. Baked Milk- and Egg-Containing Diet in the Management of Milk and Egg Allergy. Journal of Allergy and Clinical Immunology. 2015;1:13 - 23.

\section{Hosted file}

Table 1.pdf available at https://authorea.com/users/406391/articles/517068-graded-homeintroduction-of-egg-is-safe-well-received-and-cost-effective-when-managing-childhoodegg-allergy 